\title{
Comparison of Methods for Detection of Chlamydia trachomatis in Cervical from Women in Mosul City
}

\author{
Modruka M.H. Al-Jammally ${ }^{1 *}$; Hiyam A. I. Al-Taii ${ }^{2}$ \\ ${ }^{1 *}$ Dep. Int. Med. Vet. Med, College of Veterinary, University of Mosul, Mosul, Iraq \\ ${ }^{2}$ Department of Biology, College of Science, University of Mosul Mosul, Iraq \\ Email: ${ }^{1 *}$ modrukaaljamaly57@gmail.com, ${ }^{2}$ hiyamaltaii@ymail.com
}

(Received December 03, 2018; Accepted July 03, 2019; Available online March 01, 2020)

DOI: 10.33899/edusj.2020.164367, (C) 2020, College of Education for Pure Science, University of Mosul.

This is an open access article under the CC BY 4.0 license (http://creativecommons.org/licenses/by/4.0/).

\begin{abstract}
:
The study included detection of Chlamydia trachomatis in the cervix of symptomatic and a symptomatic woman.

A total of $(60)$ woman aged between $(16-<45)$ years were diagnosed by the consultant of the general hospital in Mosul city during the period May until the end of December 2013.

Three endocervical swabs were collected from each woman. One of the three swabs was stained with Giemsa stain for direct examination by light microscope, and the other swabs was put in $0.2 \mathrm{M}$ sucrose phosphates transport media then inoculated in yolk Sac of embryonated chicken eggs, and the last endocervical swabs was used for detection of chlamydia by using rapid immunochromatographic card test . From each woman participated in this study $5 \mathrm{ml}$ of venous blood was collected to detect the immunoglobulin (IgG) by ELISA and indirect microimmuno flourescence test.

The result showed that C.trachomatis was presented in percentage (35\%) by Indirect microimmunoflourescence test (30\%) by ELISA (25\%) by culturing (21.6) by Giemsa stain, , (5\%) by rapid test in symptomatic and asymptomatic women.

This result showd that Indirect MicroimmunoaFlourecence is more specific to detect Chlamydia than other test while the rapid test was of no value in detection of chlamydia in endocervical swabs

Also the result showed that the percentage of Chlamydia in a asymptomatic woman such as infertility and abortion had high percentage than symptomatic woman such as vaginal discharge, abdominal pain' and ectopic pregnancy . Also the result showed that the isolation rate in age group (21-30) years was the more dominate than other age group.
\end{abstract}

Keyword: Chlamydia trachomatis , ELISA, IMIF

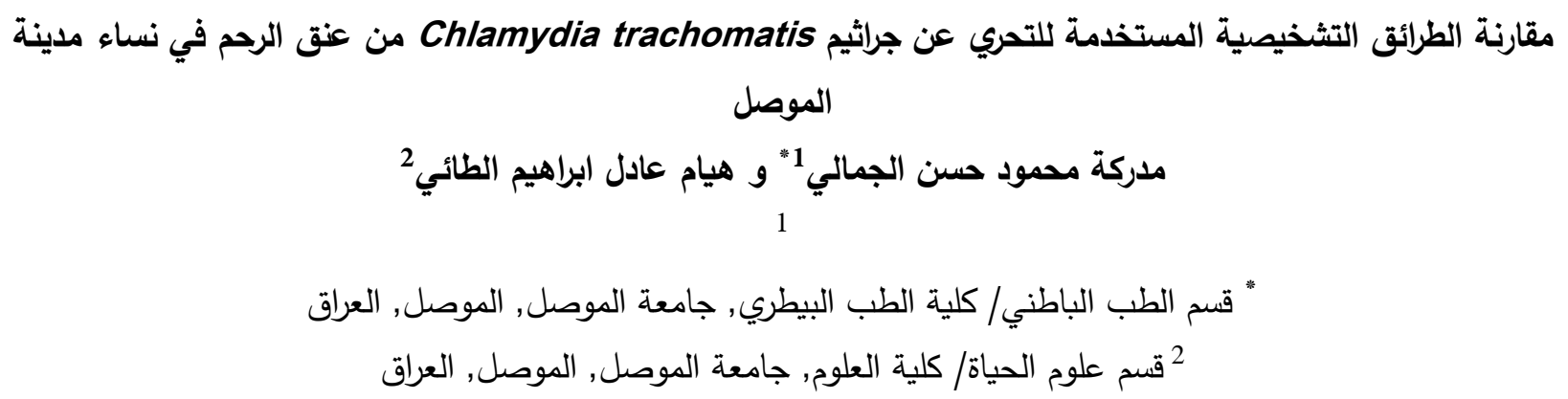


تضمنت الدراسة التحري عن جراثيم Chlamydia trachomatis في عنق الرحم للنساء الدصابات بالتهاب الجهاز التتاسلي السريري وتحت السريري. شملت الدراسة فحص (60) امرأة تراوحت اعمارهن (16-45>) سنة المشخصات سريريا من قبل الطبيبات الاختصاص في مستثفى السلام والخنساء التعليمي في الموصل وللفترة ما بين آذار حتى نهاية كانون الاول 2013.

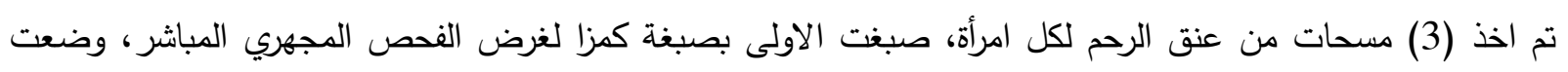

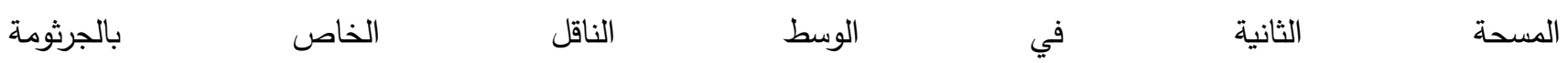
Sucrose phosphate transport medium 0.2Mالثالثة في فحص التشرب المناعي الكروماتوكرافي السريع. كما تم سحب 5 مل من الدم الوريدي لكل امرأة لغرض التحري عن الاجسام

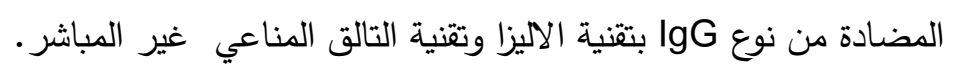

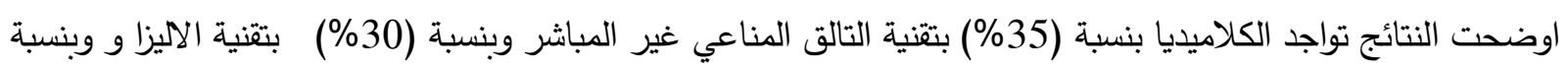

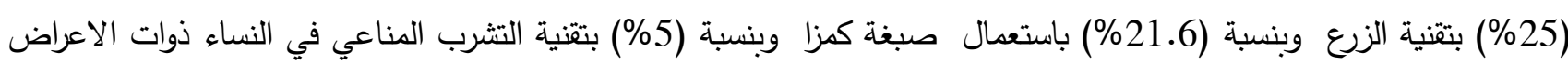

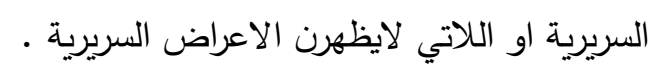
كما اظهرت الدراسة ان تقنية التالق المناعي غير المباشر تعد افضل الطرائق استعمالا في التحري عن الجرثومة في حين ان طريقة التشرب المناعي اقل حساسية ولا ينصح باستعمالها. كما اوضحت النتائج ان نسبة تواجد الكلاميديا في النساء اللواتي لايبدين اعراضا سريرية ظاهرة مثل تكرار الاجهاض والعقم

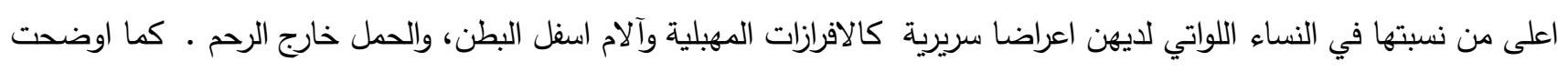
النتائج ارتفاع الاصنية لدى النساء بعمر (21-30) سنة. الكلمات المفتاحية : المتدثرة الحثرية , الاليزا , التألق المناعي غير المباشر

بكتريا صغيرة الحجم ، سالبة لصبغة كرام غير متحركة متطفلة اجباريا داخل الخلايا، تضم ثلاثة انواع مهمة للانسان وهي

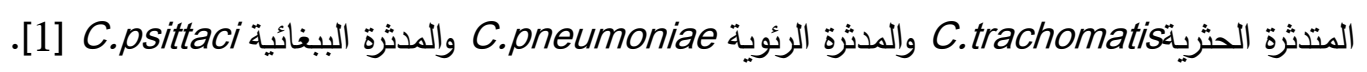

تعد بكتريا C.trachomatis اكثر الانواع انتثارا في العالم اذ تعد احدى المسببات الجرثومية المسؤولة عن الامراض المنقولة جنسيا في العالم [2]، وتثمل ثلاث انماط مصلية تحت النوع BioVars مسؤولة عن احداث امراض مختلفة في الانسان فالنمط

بسبب التهاب الاحليل والبربخ في الرجال بالاضافة إلى التهاب الرحم في النساء فضلا عن اصابته للغشاء المبطن

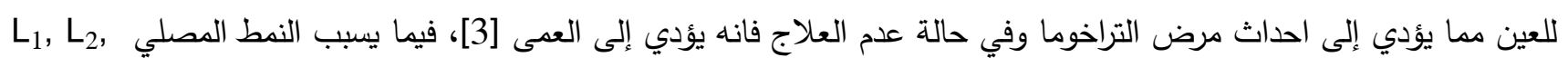

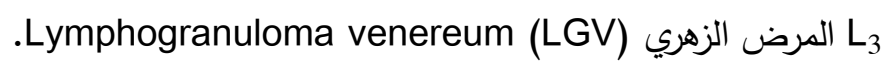

أما الانماط D-K فتسبب التهابات في منطقة الحوض Pelivic inflammatory disease [3] يطلق على معظم اصابات الكلاميديا بالمرض الصامت Silent disease إذ ان نصف إلى ثلاثة ارباع اصابات النساء والرجال تكون بدون اعراض ولهذا لا لإن 
يتم تثخيصها أو معالجتها، أما في حالة ظهور اعراض فتتمثل بالافرازات المهيلية غير الطبيعية، حرقة اثثاء التبول، طفح حول المنطقة التتاسلية وفي حالة تطور الحالة وعدم العلاج المناسب تقود إلى احداث التهاب الرحم ومن ثم تصعد الاصلية وابة وتصيب قناة فالوب مؤدية إلى التهابها وبالتالي إلى حدوث امراض تصيب المنطقة الحوضية مسببة ما يعرف بمرض الالتهاب الحوضي مما يؤدي

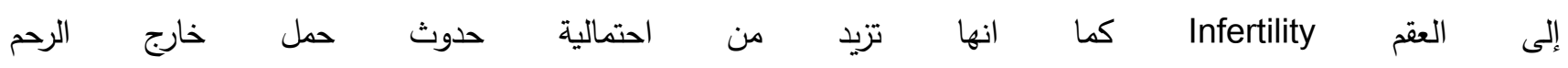
ectopic pregnancy

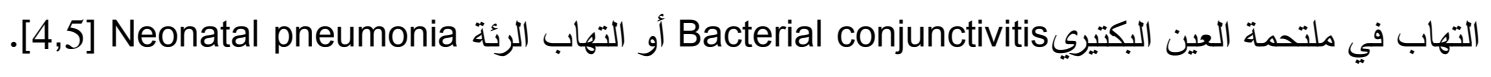

تمتلك الكلاميديا عوامل فوعة مختلفة تتمثل بقابليتها على تكوين شكلين الاول الطور المعدي Elementary body (EB) والذي له قابلية الالتصاق باسطح خلايا المضيف (الخلايا الطلائية) ومن ثم يدخل إلى داخل الخلية عن طريق عملية البلعمة Phagocytosis

الخلية مما يؤدي إلى تحطيمها [6].

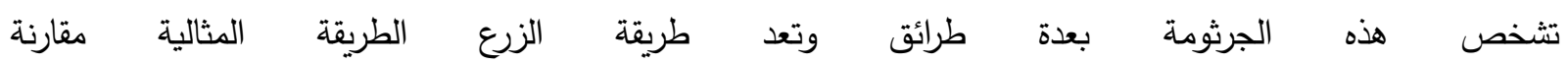

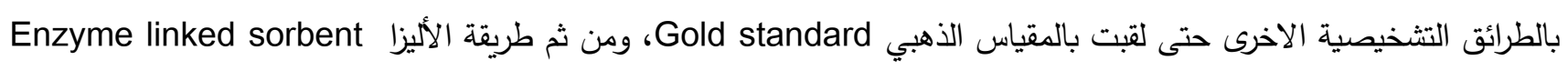
immune assay عن الحوامض النووية كتفاعل البلمرة المتسلسل (7) Polymerase chain reaction (PCR). حاولت الدراسة التحري عن هذه الجرثومة بالطرائق الزرعية والمصلية و مقارنة الطرائق المختلفة في تثخيصها. المواد وطرائق العمل

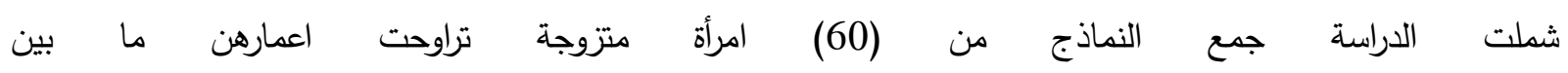
(16-45>) سنة وللفترة ما بين آذار - كانون الاول 2013.

(38) منهن يعانين من بعض الاعراض السريرية الظاهرة والمتمثلة بافرازات مهبلية، آلام اسفل البطن والظهر ، حرقة اثثاء

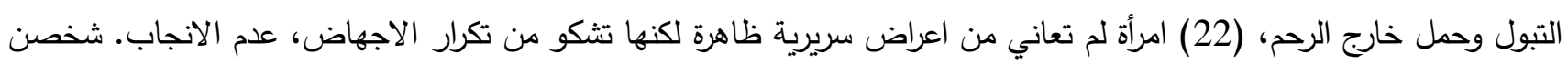

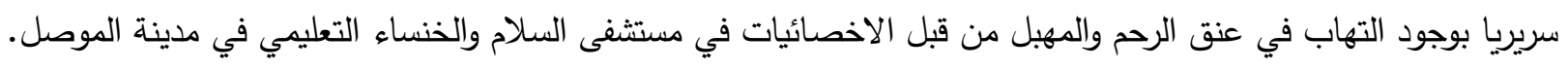
تم جمع بعض المعلومات عن كل امرأة متمثلة بالاس، العمر، السكن، عدد الاطفال، اجهاض، حمل خارج الرحم، تكرار الالتهاب. النماذج

بعد ادخال Speculum إلى داخل المهبل لتوسيع فتحة المهيل ازيلت الافرازات المخاطية الزائدة بواسطة قطن معقم. ثم اخذت دئت Special plastic shaft darcon ثلاث مسحات من عنق الرحم لكل مريضة باستعمال مسحات بلاستيكية خاصة بجراثيم الكلاميديا tip 1. فرشت المسحة الاولى بشكل دائري على شريحة زجاجية وبعد ان جفت تم تتبيتها بالميثانول لغرض الصبخ. 


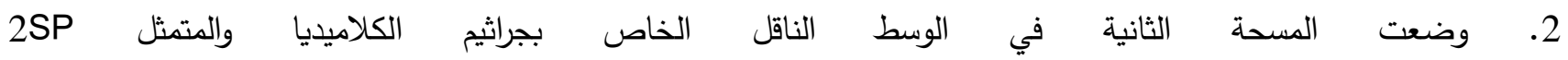
0.2M (Sucrose-phosphate transport medium) بتركيز 10 مايكروغرام/سم و Vancomycin بتركيز 25 ملغم/سم و وNystatin بتركيز 25 وحدة دولية/سم3. 3. اما المسحة الثالثة فقد وضعت في انبوبة جافة لغرض الفحص النوعي لتواجد الكلاميديا والمعروف بطريقة التشرب المناعي الكروماتوكرافي السريع. 4. تم سحب (5) مل من الدم الوريدي لكل مريضة لغرض اجراء الفحوصات المصلية. نقلت جميع العينات بالسرعة المكنة بما لا يزيد عن ساعتين إلى كلية العلوم / جامعة الموصل/ لغرض اكمال الفحوصات.

1. صبغت المسحة الاولى بصبغة كمزا الخاصة بجرثومة الكلاميديا لغرض ملاحظة الاجسام الاشتمالية [8].

2. بعد تأمين البيض المخصب من حقول خالية من الامراض وضعت في الحاضنة تم تلقيح البيوض المخصبة بعمر 5-9 أيام بالعينات الموضوعة في الوسط الناقل بنسبة (0.2) مللتر • إذ لقحت في كيس المح وغلقت الفتحة بالبارافين ووضعت البيوض

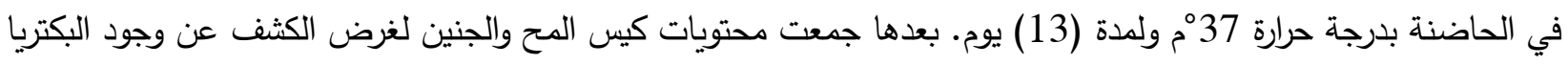
علما بأن عدد البيض المحقون لكل عزلة بيضتان مع عينة السيطرة الملقحة بالوسط الناقل بدون عينات، وقد اجريت ثلاث تمريرات متسلسلة للعزلات السالبة [9].

3. اما المسحة الثالثة فقد استعملت لغرض البحث عن مستضد الكلاميديا ضمن طريقة التشرب المناعي الكروماتوكرافي السريع Rapid immunechromatographic card test

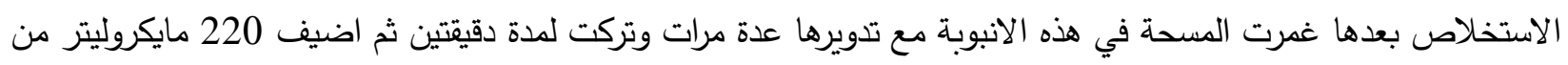

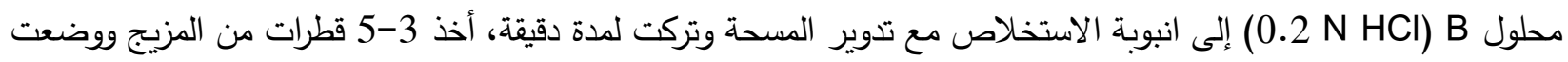
في الحفرة المؤشر عليها بحرف S (النموذج) في شريط الاختبار وسجلت النتيجة بعد 10 دقائق، وعند ظهور خطين بلون احمر

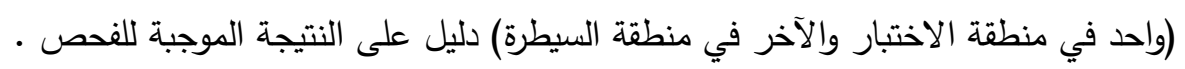

4. تم فصل المصل من عينات الدم باستعمال جهاز الطرد المركزي بقوة 3000 دورة/دقيقة لمدة (10) دقائق وحفظ بدرجة حرارة (20) (20) لحين الاستعمال.

\section{الفحوصات المصلية :}

أ. تم استعمال اختبار الاليزا كاختبار كمي للتحري عن الاجسام المضادة الخاصة بالمتدثرة الحثرية (C.trachomatis) والمتمثلة بـ والتي تعد كمؤشر للاصابة المزمنة، تم الاعتماد في قراءة النتيجة على عمل المنحنى القياسي بين (التركيز والامتصاصية)

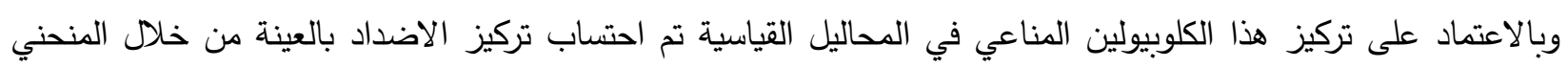
القياسي وتم اعطاء النتائج اعتمادا على القيم التالية: 
16> وحدة دولية النتيجة (سالبة)

Borderline 16 >22 وحدة دولية النتيجة مشكوك بها

22

ب. تم استعمال طريقة التألق المناعي غير المباشر Indirect Microimmuneflorescence kit (Euroimmun) للتحري عن الاجسام المضادة وgG وهو اختبار كمي ومؤشر أيضاً عن الحالة المزمنة وتعد النتيجة موجبة في حالة وجود الاجسام المضادة

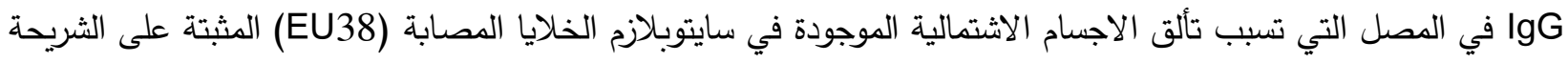

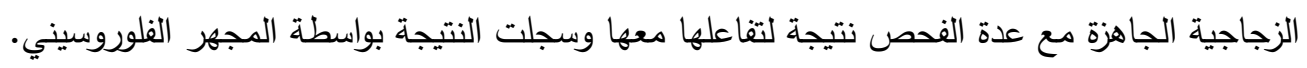

اظهرت نتائج التحري عن جراثيم C.trachomatis وبالاعتماد على صبغة كمزا المباشرة. ان عدد العينات الموجبة (13) عينة وبنسبة (6. 21\%) وتم الاعتماد في ذلك على تواجد الاجسام الاشتمالية والمصبوغة باللون الازرق في المسحات المأخوذة مباشرة

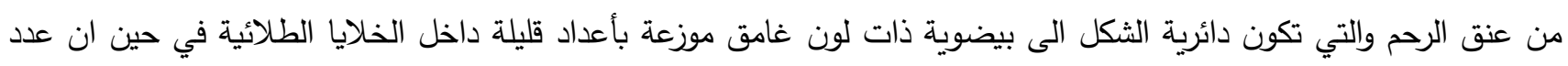



الجدول (1): اعداد ونسب حالات الاصابة بجراثيم C.trachomatis باستعمال صبغة كمزا للعينات قيد الدراسة

\begin{tabular}{|c|c|c|}
\hline النسبة المئوية & العدد & العينات \\
\hline 21.6 & 13 & العينات الموجبة \\
\hline 78.4 & 47 & العينات السالبة \\
\hline 100 & 60 & المجموع الكلي \\
\hline
\end{tabular}




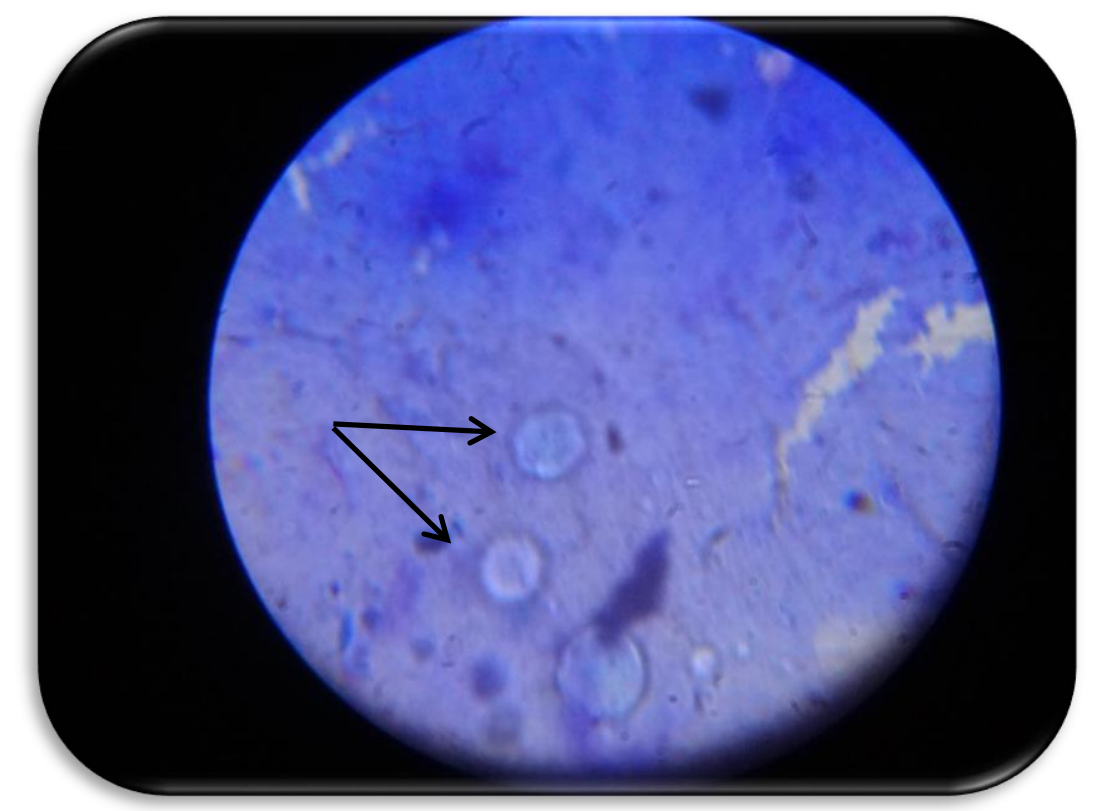

الصورة (1): الاجسام الاشتمالية للنوع المتدثرة الحثرية للمسحات المباشرة والمصبوغة بصبغة كمزا

بينت بـ C.trachomatis بلغت 15 عينة وبنسبة (25\%) في حين كان عدد العينات السالبة 45 عينة وبنسبة (75\%) وبطريقة الزرع في كيس المح لاجنة البيض المخصب وذلك من خلال ملاحظة الاجسام الاشتمالية في المسحات المحضرة من مح البيض وصبغهات البهات بصبغة كمزا بالاضافة إلى التغيرات الثكلية والفسلجية التي حدثت في كيس المح فضلا عن التغيرات المرضية التي حدثت في الاجنة

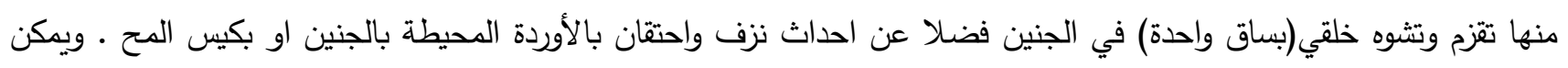
ملاحظة ذلك من خلال الصورة (2) والجدول (2).



الصورة (2): التغيرات المرضية في جنين البيض المخصب والمصاب بالنوع المتدثرة الحثرية 
الجدول (2): اعداد ونسب حالات الاصابة بجراثيم C.trachomatis في العينات قيد الدراسة وبطريقة الزرع في اجنة الدجاج

\begin{tabular}{|c|c|c|}
\hline النسبة المئوية & 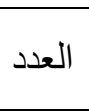 & $\begin{array}{l}\text { الاصابة بجر اثثم } \\
\text { C.trachomatis }\end{array}$ \\
\hline 25 & 15 & الحالات المصابة \\
\hline 75 & 45 & الحالات غير المصـابة \\
\hline 100 & 60 & المجموع \\
\hline
\end{tabular}

\section{التشخيص المصلي}

تم الاعتماد على ثلاث طرائق للتحري عن جرثومة C.trachomatis:

1. التحري عن وجود المستضد الخاص بالكلاميديا في المسحات المأخوذة من عنق الرحم وبالاعتماد على الطريقة السريعة والمعروفة بطريقة التشرب المناعي الكروماتوكرافي السريع كطريقة نوعية وسريعة. 2. اختبار الاليزا للتحري عن الاجسام المضادة (lgG) في مصل المريض. 3. اختبار التألق المناعي غير المباشر للتحري عن الاجسام المضادة (lgG) في مصل المريض.

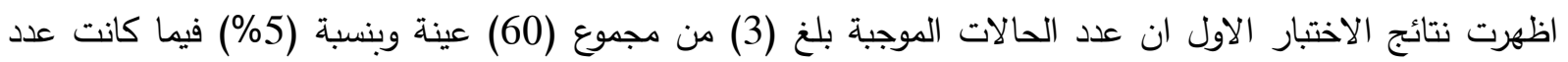
الحالات السالبة (57) عينة وبنسبة (95\%).

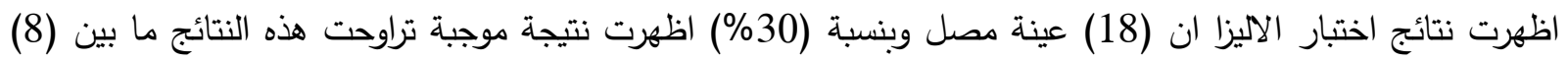

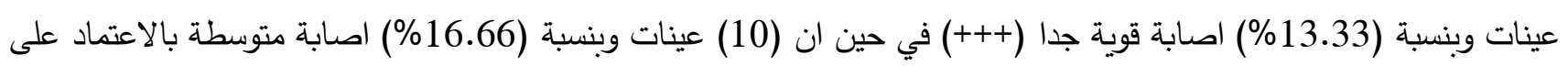
القوة الاختزالية للانزيم والتي تتناسب طرديا مع كمية الاضداد الموجودة في العينة متمثلة بشدة الالوان (++) كما ان هناك حالات مشكوك بها ويمكن ملاحظة ذلك من خلال الجدول (4).

الجدول (3): اعداد ونسب تواجد الاجسام المضادة IgG في مصل النساء قيد الدراسة وباستعمال طريقة الاليزا

\begin{tabular}{|c|c|c|}
\hline$\%$ & الاعداد الموجبة لتواجد الاجسام & نوع الحالة \\
\hline$(13.33)$ & 8 & اصلابة موجبة قوية (+++) \\
\hline 16.67 & 10 & اصLابة موجبة (+++) \\
\hline 13.33 & 8 & اصابة مشكوك بها \\
\hline 56.67 & 34 & اصابة سالبة \\
\hline 100 & 60 & المجموع \\
\hline
\end{tabular}

اظهرت نتائج تتنية التألق المناعي غير المباشر ان عدد العينات الموجبة بلغت 21 وبنسبة (35\%) تراوحت ما بين 18 (5) حالة اصابة قوية وبنسبة 30\% و3 وبنسبة (5\%) حالات اصابة ضعيفة وكما يوضح الجدول (4). 
الجدول (4): اعداد ونسب الاصابة في النساء قيد الدراسة وحسب تقنية التألق المناعي غير المباثر

\begin{tabular}{|c|c|c|}
\hline$\%$ & الاعداد الموجبة لتو اجد الاجسام & نوع الحالة \\
\hline 30 & 18 & اصلابة موجبة قوية (+++) \\
\hline 5 & 3 & اصلابة موجبة (+) \\
\hline 65 & 39 & اصابة سالبة \\
\hline 100 & 60 & المجموع \\
\hline
\end{tabular}

كانت اعداد النساء اللواتي اظهرت اعراض سريرية 5 وبنسبة (8.33\%) من العدد الكلي في حين ان عدد النساء اللواتي لم يظهرن اي اعراض سريرية وظهرت لديهن اصابة 8 وبنسبة (13.33\%) من العدد الكلي وباستعمال صبغة كمزا، اما في طريقة الزرع في اجنة الدجاج الدخصب فكانت اعداد النساء اللواتي اظهرت اصابة ولديهن اعراض سريرية ظاهرة 9 وبنسبة (15\%) في حين ان عدد النساء اللواتي لم يظهرن اي اعراض سريرية ولديهن اصابة 6 وبنسبة (10\%).

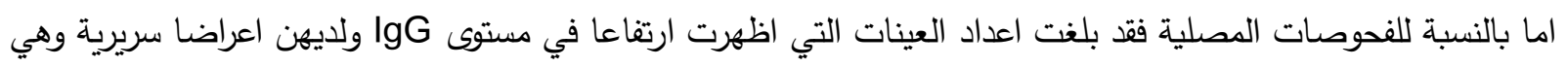
10 وبنسبة (16.66\%) في طريقة اليزا و8 وبنسبة (13.33\%) بطريقة التألق المناعي في حين بلغت اعداد النساء اللواتي لم يظهرن اعراض سريرية ولديهن ارتفاعا في مستوى IgG هي 8 وبنسبة (13.33) بطريقة الأليزا و13 وبنسبة (21.66\%) بطريقة التألق

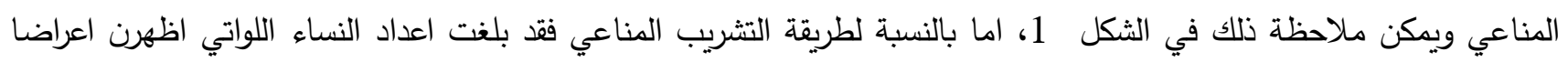

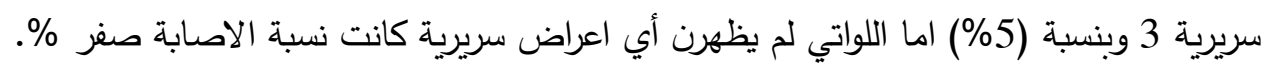

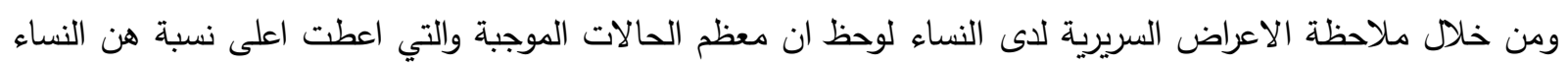
اللواتي يثكين من افرازات مهبلية تلتها آلام اسفل البطن ثم حمل خارج الرحم اما الاعراض السريرية غير الظاهرة فكانت متمثلة بعدم الانجاب وتكرار الاجهاض ويمكن ملاحظة ذلك في الثكلين (2) و (3). ولتحديد علاقة الاصابة مع اعمار المصابات فقد تم تقسيم المرضى الى اربع فئات عمرية وقد حصلت الفئة العمرية (2130) سنة اعلى نسبة وبالطرائق المختلفة فيما اعطت كل من الفئات (16-20) و (31-40) سنة نفس النسبة تقريبا وبالطرائق المختلفة

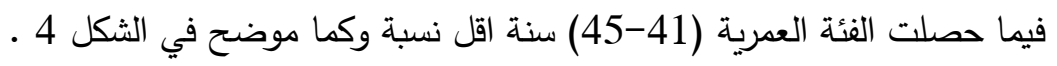

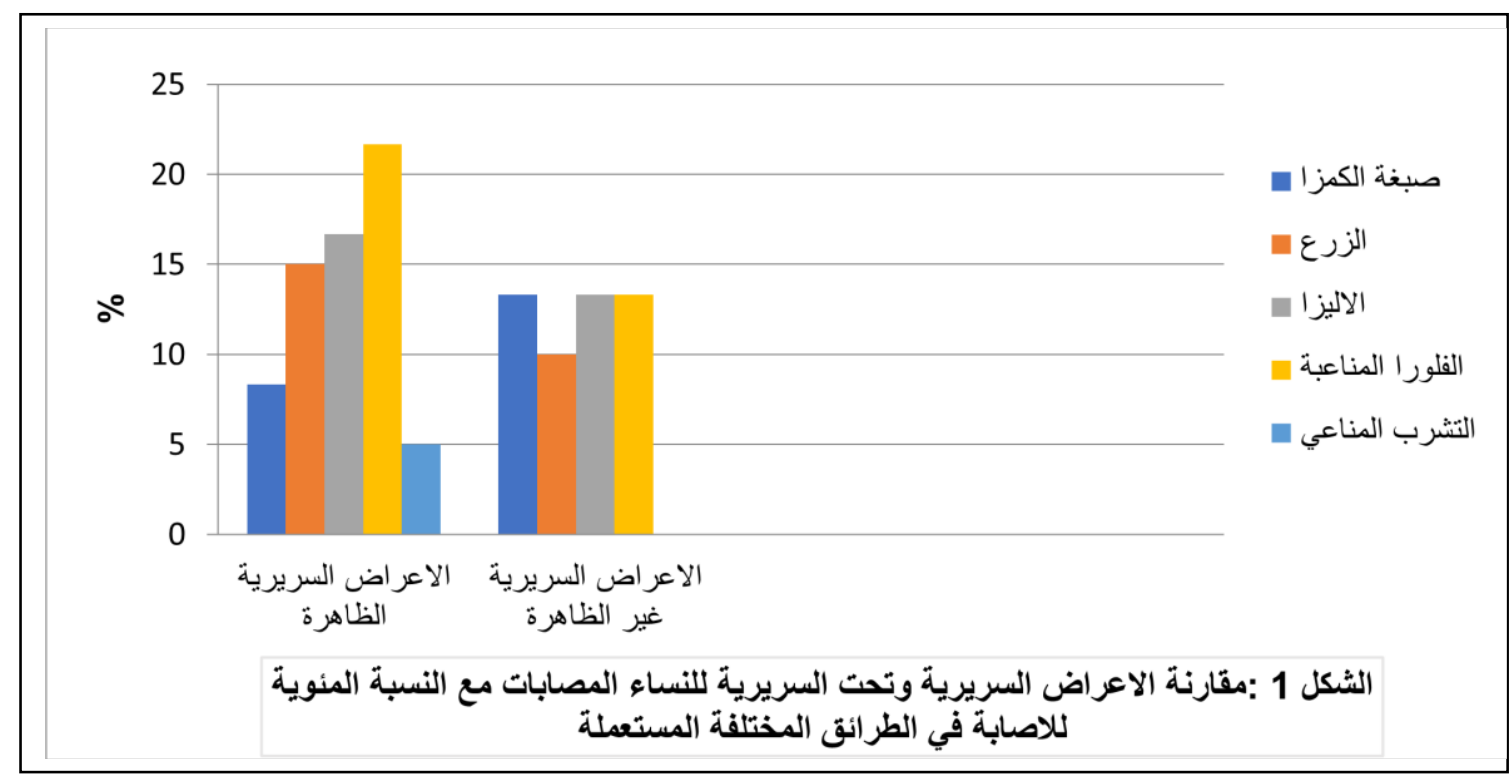



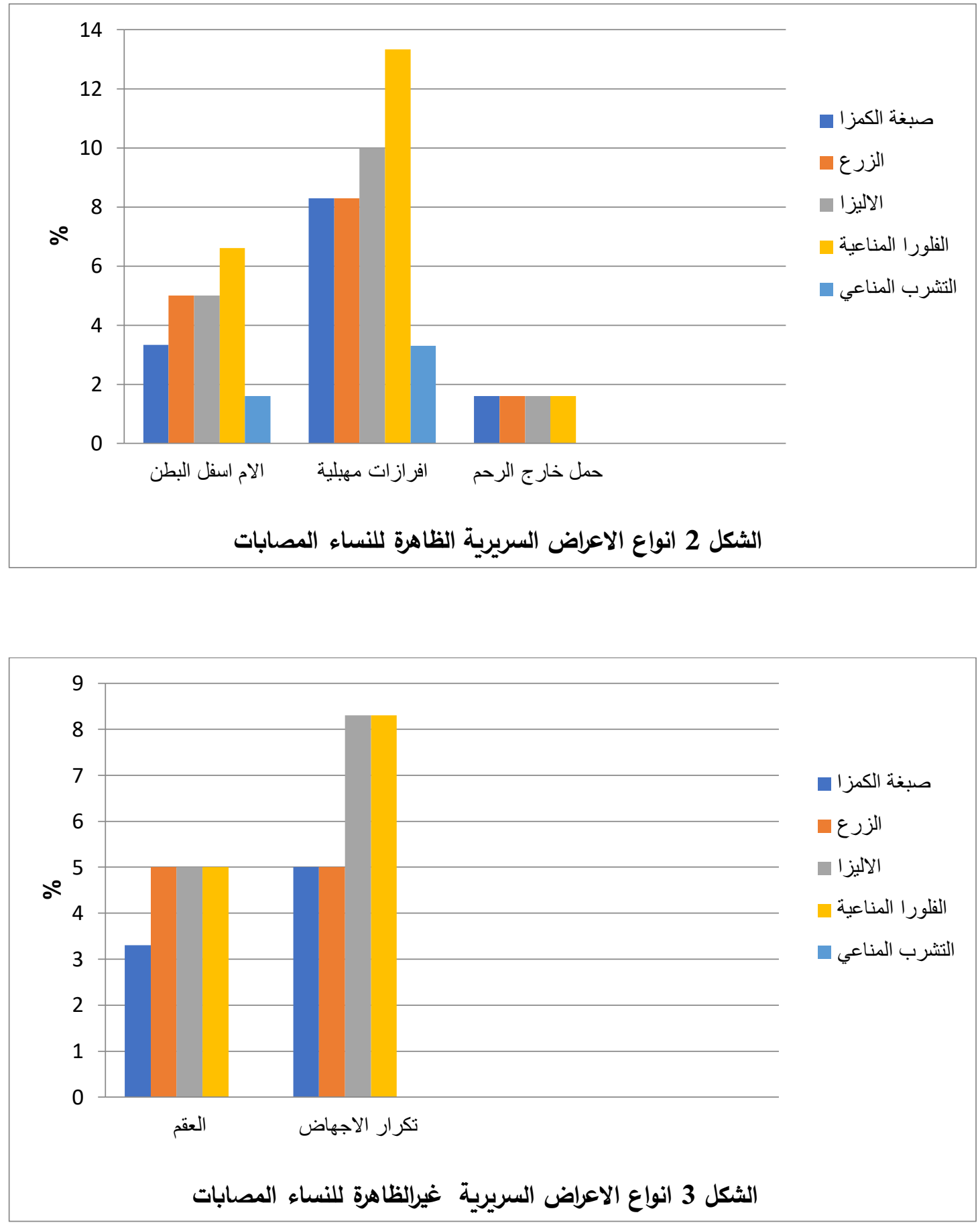


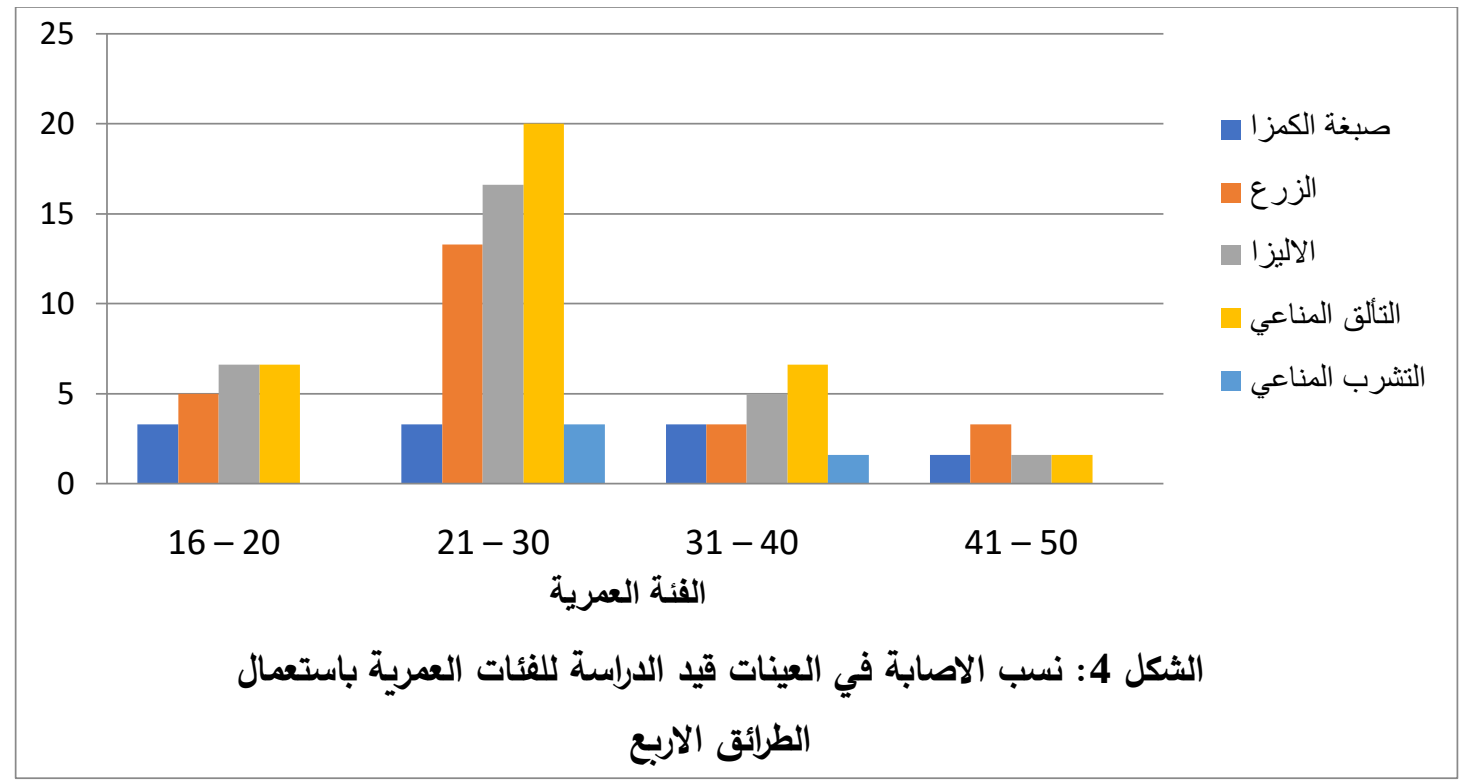

مقارنة الطرائق التشخيصية المستعملة تم الاعتماد في تثخيص جرثومة المتدثرة الحثرية C.trachomatis على الطرائق المذكورة سابقا واظهرت النتائج بان افضل

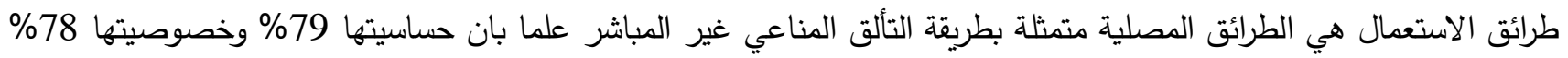
تليها طريقة الاليزا ويمكن ملاحظة ذلك من الثكل التالي :

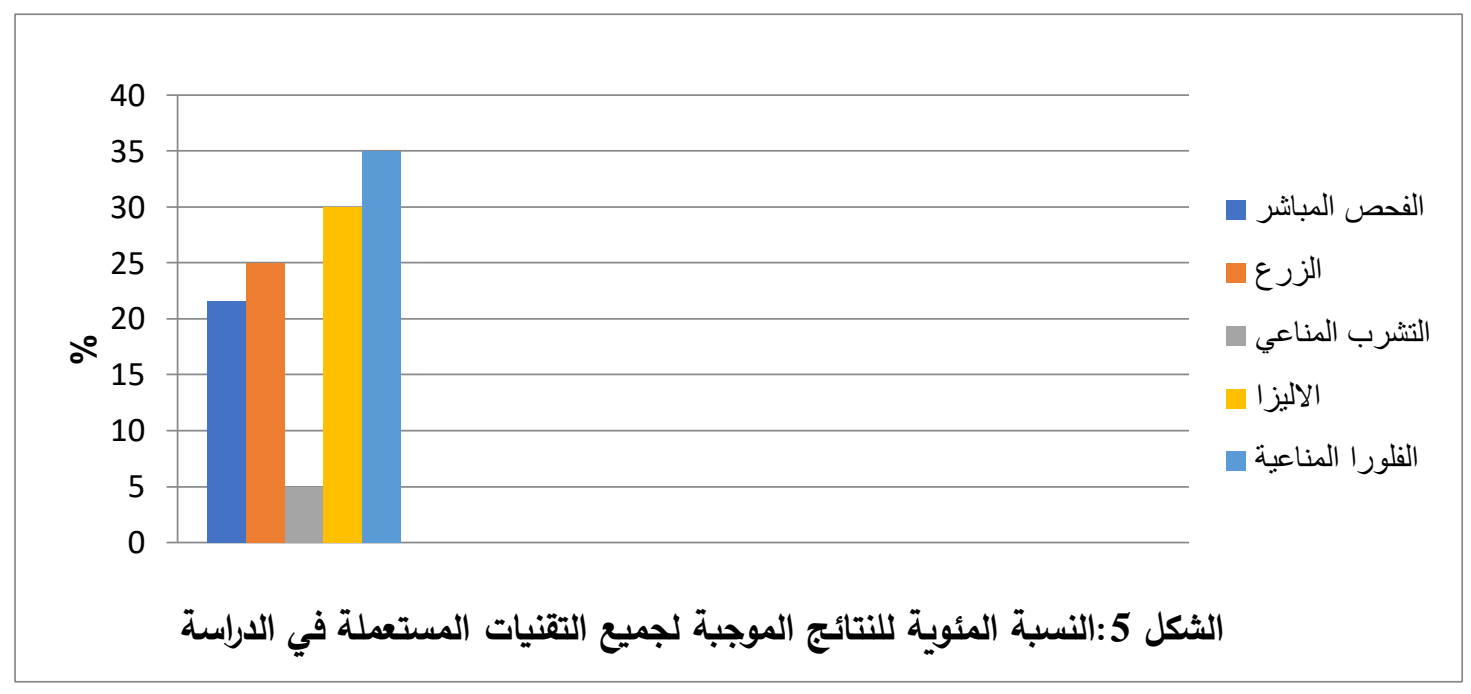

المناقثة

اظهرت نتائج التحري عن جرثومة C.trachomatis في العينات قيد الدراسة وبالاعتماد على المسحات المأخوذة من عنق الرحم والمصبوغة بصبغة كمزا، ان عدد النساء المصابات بلغ 13 امرأة وبنسبة (21.6\%) سواء كانت الاصابة ظاهرة الاعراض ام لم تكن في حين ان عدد النساء غير المصابات بلغ 47 امرأة وبنسبة (78.3\%). اعتمد في تثخيص ذلك على ملاحظة وجود الاجسام الاولية والتي تكون دائرية الثكل الى بيضوية ذات لون بنفجي غامق موزعة باعداد قليلة داخل الخلايا الطلائية. 
تعد الاجسام الاولية احد المميزات المهمة التي تتصف بها هذه الجرثومة لكونها مجبرة العيش داخل الخلايا الحية للكائنات حقيقية النواة لذلك اطلق عليها Intracellular parasite، تتواجد هذه الاجسام داخل فجوات ضمن الخلية وتتضاعف داخل الخلية لكنها تتحول الى الثكل المعدي خارج الخلية [10]. وعلى الرغم من استعمال صبغة كمزا للتحري عن الاجسام الاولية منذ قديم الزمان لكن لا تعد هذه التقنية من التقنيات الدقيقة في الكثف عن هذه الجرثومة وذلك لاحتمالية اختفاء الجرثومة ضمن خلايا الرحم. فضلا عن مدى حساسيتها القليلة اذ تثخص فقط 15\% من الكلاميديا المشخصة بطرائق اخرى كالزرع وبذلك اتفت الدراسة مع دراسة الباحثين Shaw وآخرون [11] و Lanjouw وآخرون [12].

وبما ان الصبغة المباشرة لا تكفي لتثخيص الكلاميديا لذلك اعتمد على الطرائق الاكثر تطورا والمتمثلة بطريقة الزرع في اجنة الدجاج التي تعد من الطرائق المثالية للتشخيص اذ يلاحظ من الجدول 2 زيادة اعداد المصابات وبما ان هذه الجرثومة لا تستطيع النمو إلا داخل الخلايا الحية لذلك فقد تم تتميتها في البيض المخصب [13] واعتمد في تثخيصها على التغيرات المرضية التي تحدثها في الجنين كتشوه وتقزم وتأخر النمو بالاضافة الى موت الجنين بعمر ما بين (3-14) ايام بعد الحقن فضلا عن احداث نزف واحتقان الاوردة المحيطة بالجنين او بكيس المح كما ان مح البيض يتحول إلى سائل خفيف يختلف عن المح الطبيعي بالاضافة الى تواجد الاجسام الاولية في المسحات المأخوذة من المح بعد صبغها بصبغة كمزا وبذلك تكون نتائج الدراسة قد تأكدت مع دراسة الباحث [14torze Shanna وآخرون[15] ان اكثر من 50\% من اوضح الاجنة تعاني تآخر في النمو وتتزم مع احداث نزف بالاضافة الى التواء في اصابع القدم مع احداث تثوهات خلقية للجنين، وعلى الرغم من ان طرائق الزرع تكون حساسة جدا الا انها تحتاج الى جهد شديد بالاضافة الى انها مكلفة كما انها تحتاج الى وقت طويل سواء كان الزرع في البيض المخصب ام في المزارع النسيجية فضلا عن صعوبة الحفاظ على حيوية الكائن المجهري خلال النقل او خلال الخزن [16,17].

\section{الطرائق المصلية}

اعتمد في تثخيص الكلاميديا على عدد من الطرائق المصلية متمثلة بالطريقة السريعة والمعروفة بطريقة التشرب المناعي الكروماتوكرافي السريع إذ تتحرى هذه الطريقة عن مستضد الكلاميديا في المسحات المأخوذة من عنق الرحم إذ اظهرت نتائج هذه الدراسة ان العينات الموجبة بلغت 3 عينات وبنسبة (5\%) وبذلك تنطبق الدراسة مع ما ذكره كل من الباحثين Gibneyوآخرون [18] و Morre وآخرون [19] ، لكنها اختلفت عن دراسة الباحثة Al-Ghurairi [20] ، اذ حصلت على نسب اعلى فيما لم تحصل الباحثة Mammani اخذ العينة وعلى الرغم من ان هذا الاختيار يعبر عن تواجد مستضد الكلاميديا في العينة سواءً اكانت حية أو غير حية الا ان نجاح هذا الاختبار يعتمد على عدد الكائن المجهري الموجود في العينة والتي تتأثر بطريقة جمع العينة كما انها تتأثر بعوامل تتعلق بالمريضة مثل العمر تاريخ تواجد الامراض الانتقالية، تواجد الاعراض، كما ان تواجد الكلاميديا يختلف حسب النمط المصلي [16]. في حين اظهرت نتائج هذه الدراسة بالاعتماد على الاختبارات المصلية والمتمثلة باختبار الاليزا والتألق المناعي غير المباشر لهري نسبة (30\%) و(35\%) على التوالي وقد اتفقت الدراسة مع دراسة Mammani وآخرون [21] التي اجريت في دهوك إذ بلغت 11.3 و 11.3 
يتضح من الثكل (2 و 1) ان نسبة الاصابة في النساء اللواتي اظهرت اعراضا سريرية (15.33)(15)(16.66)(21.66) في كل من صبغة كمزا والزرع والاليزا والتألق المناعي على التوالي في حين ان نسبة الاصابة في النساء اللواتي لم يظهرن اعراضا سريرية وبنفس الطرائق السابقة نفسها بلغت (13.33)(10)(13.33)(13.33) على التوالي وبذلك تكون الدراسة متفقة مع دراسة Verkooye وآخرون [22] إذ وجد ان 1.7-10\% من النساء لا يظهرن أي اعراض مرضية لتواجد الكلاميديا لكنهم اظهروا فحصا موجبا لتواجدها.

تكمن خطورة الكلاميديا في حالة عدم ظهور الاعراض ولذلك اطلقوا عليها بالمرض الصامت Silent Disease إذ لا يمكن تشخيصها ولا يمكن علاجها مما يؤدي إلى صعود الاصابة إلى الاعلى واحداث تعقدات مرضية كانسداد قناة فالوب واحتمالية حدوث حمل خارج الرحم [10].

معظم حالات الاصابة ظهرت في النساء اللواتي يعانين من افرازات مهبلية والالام في البطن وحمل خارج الرحم وتعد هذه اهم

الاعراض التي تظهر عند الاصابة بهذه الجرثومة وبذلك اتفقت مع دراسة الباحثة Mammani وآخرون [21]. معظم النساء اللواتي لم يظهرن اعراضا سريرية واضحة ولديهن اصابة كن ممن يعانين من تكرار الاجهاض بنسبة 5\% في كل من صبغة كمزا وتقنية الزرع و8.3\% بتقنيتي الاليزا والتألق المناعي غير المباشر اما العقم فقد كانت النسبة 3.3\% بصنبة كمزا و5\% في كل من تقنية الزرع والاليزا والتألق المناعي غير المباشر • وقد اشارت دراسة Malik وآخرون [23] ان 36.2\% من النساء اللواتي لم يظهرن اعراضا كن يعانين من العقم. اشار الباحث Malanie وآخرون [24] ان 27.7\% من النساء اللواتي لا يظهرن اعراض كن ممن يعانين من تكرار الاجهاض وان ظهور هذه النتائج الموجبة تؤكد اهمية استعمال هذه التقنيات المصلية. وعند مقارنة نسب الاصابة في الفئة العمرية يتضح من الثكل 4 ان اعلى نسبة اصابة كانت بالفئة العمرية 21-30 سنة واقل نسبة في الفئة العمرية 41-50 سنة وبذلك تكون الدراسة متفقة مع دراسة Mammani وآخرون [21]. تحدث الكلاميديا بشكل رئيس في النساء بعمر اقل من 25 سنة وبنسبة اعلى من 30\% وخصوصا في النساء النشيطات جنسيا إذ ان الطبقة الطلائية لمنطقة المهيل لغير البالغة تكون عرضة للاصابة [25,26]. اظهرت نتائج الدراسة الحالية بان افضل طريقة للاستعمال هي طريقة التألق المناعي غير المباشرة تلتها طريقة الاليزا ثم الزرع واقلها هي طريقة التشرب المناعي الكروماتوكرافي السريع وبذلك تكون الدراسة متفقة مع دراسة Sweet [27]. وعلى الرغم من ان تقنية الزرع هي افضل الطرائق لانها تؤكد وجود الكلاميديا الحية القابلة للتكاثر الا ان حساسيتها وخصوصيتها تقل عن الطرائق المصلية. كما ان طريقة التألق المناعي اكثر تخصصية في التحري عن الكلاميديا [28]. اذ اظهرت النتائج ان نسبة تواجد الكلاميديا في طريقة التألق المناعي اعلى نسبة من الطرائق الاخرى.

المصادر

1. Smith, I.W. (2010). Chlamydia In Greenwood, R.; Slack, R. and Peether, J. (ed.). Medical Microbiology $5^{\text {th }}$. Edition, Churchill living stone, London., 361-367.

2.Sachse , k., Bavoil, p.m.,kaltenboectk., B., Stephens . R.S.,Kuo.CC., Rossello _Mora , R., Horn.M., (2015) Appl Microbiol., 38(2) : 99_103 
3. Zigangirova, N.A.; Rumyatseva, Y.P.; Morgunova, E.Y.; Kapotina, L.N.; Diolenko, L.V.; Kost, E.A.; Koroleva, E.A.; Bashmakov, Y.K. and Petyaev, I.M. (2013).., Bio. Med. Research International.10: 486- 489

4. Black, C.M. (2013).. Ameri society of Microbs .26 : 536 - 541

5. Lanjouw , E ; Oubury, S; Devries . H.J., Stary , A., Radeliffe,K.(2016) . international Jouranal of STD \& AIDs 27(5) : 333-438

6. Petrovay , F., Balla,E.,Erdosi,T.(2017) Euro Surveill 2 ; (22) - (5) 30-45

7. Estam, M. (1998). Chlamydia infection. In Braanwald, E. Fauci As Kasper Dl et al (ed.) Harrison of Internal Medicine $15^{\text {th }}$. Mc Graw Hills medical publishing divison., pp.1075-1082.

8. Brooks, G.F.; Batel, J.S. and Morse, S.A. (2004). Chlamydia. Medical Microbiology, $22^{\text {nd }}$. Lange Medical Books/Ms Graw-Hills,pp.306-314.

9. Cruickshank, R.; Duguid, T.P.; Marmion, B.P. and Swain, R.H. (1975). "Medical Microbiology". Churchill, Livingstone. England.

10.Babinska , I.,Halanova ,M.;Kalinova, Z. ; Cechova,L.;Cislakova,L. and Geckova,A.M.;( 2017 ). Interntional J. of Enviromental Research and Puplic Health.(14).1579.

11. Shaw El, Dooley CA, Fischer ER, Scidmore MA and Field KA et al (2000). Mol. Microbial 37: 913- 925.

12.Lanjouw , E. ; Ouburg, S. ; Vries,H. J. ; Stary, A. ; Rdcliffe ,K. and Unemo , M. , (2015) International J. of STD \& AIDs, : 1-16

13. Celebi, B.S. \& Seyyal, A.K. (2006). Avian Dis., 50:489-499.

14. Storze, J. (1971). Chlamydia and Chlamydia induced disease-spring field III inesis. Charles Thomas publisher.

15. Khanna, R.N.S.; Grpta, R.K.P.; Purogit, V.D. and Sadana, J.R. (1987). Int. J. Anw. Sc.; 57(2):121123.

16. Schachter, J. (1982). Postgraduate Medicine, (72):60-69.

17. Forbes, B.A.; Sahm, D.F. and Weissfeld, A.S. (2005) Balley and Scotts Diagnostic Microbiology $.11^{\text {th }}$.ed. Mosby, Inc. In: Philadelphia, DA. USA. Pp. 572-580.

18. Gibney, L.; Mascaluso, M. and Kirk, K., (2001). Trans. Inf. 77:344-350.

19. Morre SA, Karimi O, Oubury S. (2009). Med Microbial; 55: 140 -153.

20. Al-Ghurairi A.Z. ( 2005 ) Thesis .University of Mosul . College of Medicin

21. Mammani, I.M.; Goreal, A.A.; Omer, W.J. and Mansor, S.A. (2012). China Medical Science. (9)(2). pp.112-116. 
22. Verkooye, R.P. Peeters, M.F. VonRissoort-vos, J.H.; Van der Meijden, W.I. and Mouton, J.W.; (2002). Int. J. STP. AID. 23-25.

23. Malik, A.; Jain, S.; Hakim, S.; Shukal, I. and Rizvi, M. (2006). Med. Res. (123): pp.770-775.

24. Malanie, R. Joshi, P.S. and Mathur, M.D. (2006). Medical Microbiol. 24. 2 , pp.97-100.

25. Robert, E.; Wlber, J. John, R.; Joan, S. and Richard, S. (2002). National Center for Infectious Disease. (51). (15): RR-15.

26.Ceovic , R. and Jerkovic Gulin, S. (2015) . Infect Drag Resistent .(8) : 39-47

27. Sweet, R. L.(2012) . Dis .Rep.(14):194 -203.

28.Nwokolo , N.C. ; Dragovic , B. ; Patel . S. , (2015) . International Journal of STD \& AIDs 2016 , 27(4):251-267. 